Meta

Journal des traducteurs

Translators' Journal

\title{
L'effleurement, la trace ou le renoncement
}

Un traducteur répond à U. N. Singh

\section{Jean-Claude Gémar}

Volume 39, numéro 2, juin 1994

La traduction vue de l'extérieur - Translation: a view from the outside

URI : https://id.erudit.org/iderudit/002848ar

DOI : https://doi.org/10.7202/002848ar

Aller au sommaire du numéro

Éditeur(s)

Les Presses de l'Université de Montréal

ISSN

0026-0452 (imprimé)

1492-1421 (numérique)

Découvrir la revue

Citer cet article

Gémar, J.-C. (1994). L'effleurement, la trace ou le renoncement : un traducteur répond à U. N. Singh. Meta, 39(2), 404-407. https://doi.org/10.7202/002848ar d'utilisation que vous pouvez consulter en ligne.

https://apropos.erudit.org/fr/usagers/politique-dutilisation/ 


\section{L'EFFLEUREMENT, LA TRACE OU LE RENONCEMENT. UN TRADUCTEUR RÉPOND À U. N. SINGH}

JEAN-Claude GÉMAR

Université de Montréal, Montréal, Canada

La langue est le creuset où se sculpte l'histoire d'un peuple. Les mots qui la composent et le discours qui l'exprime traduisent les valeurs du patrimoine commun que chaque génération s'efforce de transmettre. Que la suivante n'a de cesse de revoir, d'interpréter et de corriger selon les critères et les mœurs du temps. Pour U. N. Singh, le développement de la langue sera plus rapide si elle fait montre d'esprit d'ouverture à la traduction («translative»), au lieu de s'en tenir à sa capacité d'innovation («innovative»). On en comprend les raisons, eu égard aux processus de normalisation «primaire» et «secondaire» sur lesquels Singh appuie son argument. On sait ce que le développement des langues doit à la traduction.

Mais que la langue se développe suivant sa dynamique propre ou qu'elle le fasse en suivant le sens qui lui est indiqué, au hasard des circonstances (conquête, fait du Prince, idéologie du moment), a finalement peu d'importance. Ce qui importe est qu'elle soit, ait été ou sera. La trace en subsistera dans son sillage. L'exemple du grec, puis du latin en témoigne, si besoin était. D'ailleurs, les deux modèles coexistent parfois, dans l'espace comme dans le temps. Or, seul le temps nous permet - et encore sur le long, voire, à l'échelle humaine, le très long terme - de dire si le modèle interne l'emporte en avantages sur l'externe. Le talent d'organisation et de planification (linguistique, entre autres) des Romains prime-t-il, dans l'ordre des valeurs universelles, le génie créateur que les Grecs ont manifesté - et avec quel éclat! - dans tant de domaines ? Rien n'est moins sûr : que serait l'un sans l'autre?

L'affirmation d'une identité, la revendication d'une culture, la revalorisation d'un statut peuvent passer ou non par la traduction. Celle-ci n'est qu'un des moyens possibles, et non le seul. On sent, par simple intuition, qu'une société qui ne s'en remettrait, nolens volens, qu'à la traduction comme mode de développement (de sa langue, de sa culture, de son savoir) serait en grand danger d'y perdre son âme. Une société uniquement traduite est en déficit d'expression ( $c f$. Gémar 1983). La Pléiade, en France, et la Vulgate, en Allemagne, montrent que lorsque la traduction, qui est en soi le formidable vecteur d'enrichissement que l'on sait, est combinéc avec d'autres moyens qui la complètent ou en corrigent les excès, le résultat, quelque imprévisible qu'il soit, peut dépasser toute attente. Sur ce plan-là et à ces conditions, la traduction est probablement, pour une langue, un moyen plus rapide et plus simple de se développer (il suffit d'adapter à la réalité locale un modèle conçu ailleurs) que de compter sur une capacité d'innovation toujours aléatoire. La «force» d'une langue réside-t-elle, comme on semble le croire communément, dans sa capacité à «repousser l'étranger»(Berman $1984: 26$ ), retranchée 
derrière les remparts de la forteresse assiégée ? Sa véritable force ne viendrait-elle pas plutôt, comme le croyait Gœthe, du fait qu'elle puisse le dévorer ?

La responsabilité du traducteur, nous dit Singh, est considérable lorsqu'il est placé devant le dilemme que lui pose la langue de départ (LD) et qu'il doit trouver un équivalent en langue d'arrivée (LA) d'un terme étranger à sa langue. Elle l'est, en effet. Empruntant ce concept aux juristes, je parle à ce sujet d'une triple obligation du traducteur (de moyens, de résultat et de garantie). La solution du néologisme, calque ou emprunt peut être vue comme un enrichissement pour la langue d'arrivée. À cet égard est-il préférable, pour marquer la «différence» et l'origine, de conserver, mettons, le mot leader tel quel, comme on le voit encore en français ou en italien des décennies après son apparition, ou de l'assimiler sans vergogne comme l'ont fait les Espagnols : (el)lider ? Question de choix culturel et de planification linguistiques, sans doute. Mais quand une langue, l'anglais par exemple, produit - au rythme vertigineux des réalisations scientifiques et techniques des Etats-Unis - du vocabulaire à raison de plusieurs dizaines de milliers de termes nouveaux par an, l'adaptation, puis l'intégration d'une telle avalanche de mots à une autre langue, a priori moins «riche», ne vont pas de soi. Elles sont pourtant nécessaires au développement de la langue et du pays d'accueil, lequel manquerait, sinon, le train du développement tout court, que son rapport à la langue de départ se situe sur un plan vertical ou sur un axe horizontal. Cet ordre quantitatif est un préalable au qualitatif, qui procède de la capacité d'innovation d'un peuple. Le temps fera le tri entre le superflu et l'indispensable, ne retenant que ce qui, pour certains, constitue un «bon» usage et, pour d'autres, un usage utile ou fonctionnel et, somme toute, sélectif. Faute d'un tel transfert entre vases communicants, la langue à développer, appauvrie, se sclérose. À terme, elle risque de disparaître. Aussi des facteurs tels le volume du vocabulaire et la rapidité d'absorption (d'assimilation ?) doivent-ils être relativisés dans le lent processus d'évolution d'une langue par rapport à ses origines (plus ou moins) lointaines.

$\mathrm{Si}$, de tels rapports, devait découler une théorie de la traduction fondée sur un axe horizontal, comme le pense Singh, de préférence au vertical, cette théorie ne représenterait, à mon avis, qu'un «fragment» du versant d'une réalité dont la complexité passe encore l'entendement humain. Toutes les langues (les occidentales, en tout cas) sont passées, à un moment précis de leur histoire, par le stade vertical, celui où l'influence s'exerce de haut en bas, dans une situation du type DOMINANT $=\Rightarrow$ dominé. En position de dominé au départ, elles se sont progressivement (ou brutalement, selon les cas) libérées du joug sous lequel les maintenait une tradition ( $c f$. roman et germanique contre latin, en 842) ou une occupation étrangère ( $c f$. anglais contre français, français contre allemand et anglais; allemand contre français ou suédois; flamand contre espagnol; espagnol contre arabe, etc.). Elles sont sorties - temporairement du moins - victorieuses de cette confrontation, enrichies et renforcées et se situent, pour la plupart, dans un rapport horizontal du type LD $\longrightarrow$ LA. Dans cette opération, la traduction a joué, de toute évidence, un rôle déterminant, exemplaire, mais non le premier ni le seul. Ce sont des hommes tels Charlemagne ou Luther, saint Augustin ou Dante, Chaucer ou Rabelais qui ont contribué à faire changer les mentalités. Transposée à notre époque, et sur ce point je rejoins Singh, cette situation tend vers la relation horizontale, du moins entre les principales langues véhiculaires, le cas de l'anglais, lingua franca de cette fin de siècle, représentant un cas à part : dans ses rapports bilatéraux avec la langue de Shakespeare (de «Mac Do» ou de Walt Disney serait plus approprié...), toute langue, d'emblée infériorisée, est placée en relation verticale. C'est que le marché, celui de l'édition particulièrement, dicte sa loi, en traduction comme ailleurs. Je dis bien tend vers la relation horizontale, car la réalité, toujours plus nuancée, est fort différente. Ce ne sont pas les grandes puissances - littéraires, économiques, militaires ou commerciales — qui 
produisent, toutes proportions gardées, le plus de traduction, mais les «petits» pays : Belgique, Finlande, Pays-Bas, Norvège, etc. ( $c f$. Index Translationum, Unesco). Dans leur cas, nécessité fait toujours loi.

Cela dit, la relation horizontale est, en ces jours caractérisés par les mouvements de libération et d'affirmation des droits individuels, la norme du fondement consensuel de la traduction, sa justification et son honneur. La diversité est le garant, voire le siège de l'égalité (Jacquard 1978; Serres 1991). Il subsiste néanmoins des reliquats irréductibles de situation verticale, laquelle n'a pas brûlé, loin s'en faut, ses derniers feux. C'est qu'elle est inscrite au plus profond de l'être et, pour l'éradiquer, il faudra d'abord que l'humanité s'arrache des fers de ses origines, car le comportement social de l'être humain, contrairement à ce que beaucoup croient, procède non pas du langage mais de «la vie et la survie sur les hauts plateaux d'Afrique orientale» (Reichholf $1991: 218$ 219). Peut-être, à force de projeter son regard vers l'avenir plutôt que de le tourner vers le seul passé, pour qu'advienne enfin le présent invoqué par Octavio Paz (1990), l'être humain finira-t-il par s'accomplir? Dans cette perspective, la traduction, particulièrement lorsqu'elle s'exerce sur le plan horizontal, devrait fortement contribuer à l'y aider.

Il n'en reste pas moins qu'une théorie de la traduction ne saurait ignorer la dimension diachronique - qui est la partie immergée de l'iceberg, la plus importante — d'une activité remontant aux origines de la civilisation. Le faire reviendrait à nier le parcours accompli depuis au moins deux millénaires et à passer par dessus les deux étapes - l'état théologique et l'état métaphysique - qui ont précédé l'actuelle, celle que j'ai qualifiée de «quasi scientifique» (voir Gémar $1985: 236$ ).

Revendiquons plutôt pour la traduction une réflexion théorique qui, ignorant le «fragment», viserait la synthèse du savoir, quelque fragile et aléatoire qu'elle soit (Serres 1992 : 176). Pour cela, la quête d'une théorie de la traduction le moindrement intégrée (diachronique et synchronique) devrait nécessairement emprunter le parcours épistémologique balisé par la philosophie. D'emblée, le traductologue devra faire le choix décisif entre les «pensées de systèmes» (Glissant 1993 : 27) de la tradition léguée par Aristote, celle qui montre l'Etre immobile, et les pensées de mouvement portées par l'autre tradition, la platonicienne, celle du mouvement et du perpétuel devenir, celle de «l'Étant». La réunion des deux provoquera peut-être l'homogénéité («homogeneizing») dont parle Singh. Entendons-nous bien : il ne s'agit pas, par la recherche des universaux, d'uniformiser les langues et, ce faisant, les cultures et, finalement, les sociétés. Au contraire, la rencontre - par les universaux qu'elles ont en commun - des langues et des cultures, dans la mosaïque de leurs diversités, est la véritable source d'enrichissement de chacune d'elles.

Effleurement (la traduction n'est, au mieux, qu'une «approximation», nous dit justement Singh), certes, du texte de départ pour en interpréter le sens et les significátions (Gémar 1994). Mais, à l'arrivée, selon l'idéologie personnelle du traducteur, choix décisif entre l'Être et l'Étant, soit entre le renoncement et la trace.

\section{RÉFÉRENCES}

BERMAN, Antoine (1984) : L'épreuve de l'étranger : Culture et traduction dans l'Allemagne romantique, Paris, Gallimard.

GÉMAR, J.-C. (1983) : Les trois états de la politique linguistique du Québec. D'une société traduite à une société d'expression, Québec, Éditeur officiel du Québec, Dossiers du Conseil de la langue française, études juridiques no 17.

GÉMAR, J.-C. (1985) : «Réflexions sur la manière de traduire ou les trois états de la traduction», Meta, vol. 30-3, sept. 1985 , p. 236.

GÉMAR, J.-C. (1994) : Traduire ou l'art d'interpréter, Montréal, PUM (sous presse).

GLISSANT, Édouard (1993) : «Le Cri du monde», conférence inaugurale du Carrefour des littératures européennes de Strasbourg (4 nov. 1993), Le Monde des livres, vendredi 5 novembre 1993, pp. 28-29. 
JACQUARD, Albert (1978) : Éloge de la différence : la génétique et les hommes, Paris, Seuil.

PAZ, Octavio (1990) : La quête du présent. La búsqueda del presente, discours de Stockholm, Paris, Gallimard, édition bilingue.

REICHHOLF, JOSEF (1991) : L'émergence de l'homme, Paris, Flammarion. (Traduction française de Das Rätsel der Menschenwerdung, par Jeanne Étoré, Munich, Deutsche Verlags-anstalt, 1990).

SERRES, Michel (1991): Le Tiers-Instruit, Paris, François Bourin.

SERRES, Michel (1992) : Éclaircissements, Paris, François Bourin 